\title{
COMPARATIVE ANALYSIS OF HIGH-LIFT AIRFOILS FOR MOTORSPORTS APPLICATIONS
}

\author{
MOHAMMAD FOZAN UR RAB, MUHAMMAD JUNAID ALAM, \\ AHSON AKHLAQUE \& MAHRUKH MAHRUKH \\ Department of Mechanical Engineering, NED University of Engineering \& Technology, Pakistan
}

\begin{abstract}
The purpose of this study is to analyse various high-lift low-Reynolds-number airfoils using the XFOIL airfoil analysis code in the isolated flow field and to select the optimum airfoil to suit motorsports applications. The airfoil is selected after comparing the stall behavior, transition location, pressure recovery, pressure distribution and boundary-layer characteristics of various airfoils. The prime consideration while selecting an airfoil is the highest $\mathrm{Cl}$ while achieving the sustainable performance over a range of Reynolds numbers encountered on the race track. An increase in $\mathrm{Cl}$ is always accompanied by an increase in $\mathrm{Cd}$; however, this must be compromised since the main goal is to increase the aerodynamic grip. It is always desirable to increase the downforce in Formula One /Formula Student to gain a reduction in lap time. This paper establishes the criteria for the selection of high-lift low-Reynolds-number airfoils, while considering the various parameters that affect the performance of airfoils.
\end{abstract}

Keywords: aerodynamics, airfoil, downforce, Formula Student, lap time.

\section{INTRODUCTION}

When it comes to motorsports, aerodynamics play a significant role, whether it is in Formula Student (FS), Formula One (F1) or the Formula Society of Automotive Engineers (FSAE). In recent years, there have been strict limitations to the use of aerodynamic devices in regard to their position, size, and shape. However, the FS/FSAE are an exception to this rule, as there are no restrictions to the specifications of aerodynamic devices. The FSAE/FS is one of the largest educational engineering competitions, which invites students from different universities over the world to design, fabricate, test, and run a formula-themed open-wheeled car. The majority of FSAE teams tend to neglect the crucial importance of aerodynamics, judging by the low speeds that are encountered on the tracks of FSAE. In recent years, however, more and more teams seem to be utilizing aerodynamics for the optimization of their race cars. Aerodynamic devices were employed by all of the top three teams at FSUK 2017.

Usually in these competitions, the Reynolds number ranges from $10^{4}$ to $10^{6}$, which is termed as a low Reynolds Number [1]. Recently, aero packages have been employed in almost all F1 and FS vehicles [2]. These include a combination of different aerodynamic devices such as under-tray, sidepods, side skirts, diffusers, front and rear wings, etc. However, the front and rear wings largely contribute to the generation of downforce. This idea of generating downforce to help improve the traction was inspired by the aeronautical industry in the 1960s [3]. In aircraft, wings are used to generate enough lift to stay airborne, and the automotive industry uses the lift to increase the tractive force.

Michael May, a Swiss Engineer and race car driver, recognized the contribution of using an airfoil to generate a negative lift. This lift would, in turn, push the car towards the ground, thereby improving the grip, traction, and the handling of the vehicle [4]. He mounted a huge inverted wing over the cockpit of his Porsche Type 550. This wing was a success as it beat all other vehicles in the first race at the Nürburgring $1000 \mathrm{~km}$ race in 1956 . However, the Porsche Factory Team opposed the use of these wings and requested the organizers of 
the race ban them; they believed they blocked the view of the drivers behind the car. This ban stunted the development of downforce generation. However, the innovative idea was still pursued; in 1963, Jim Hall used an adjustable wing on his Le Mans car and won the Chaparral $2 \mathrm{E}$ to reduce drag [4]. After the success of Jim Hall and the adjustable wing, the idea gained traction and manufacturers from all around the world starting making cars wholly based around this concept. Since then, countless airfoils have been modified, designed, and tested. However, because most findings in the formula car industry remain classified, the selection criteria of the optimal airfoil remains a mystery. This study focuses on the low Reynolds Number to help find a reasonable estimate of the gain in downforce, especially while cornering on tracks.

The chief requirement when it comes to motorsports is to maximize the upper limit of the lift coefficient [5]. This is because high lateral acceleration can be achieved and sustained by an increased downforce generated by a high-lift coefficient, which directly contributes to the increase in cornering speed [6]. The main goal is to keep the wings within any limits or restraints that are imposed by the race organizers and yet still achieve a maximum downforce. This goal should be considered before any other criterion. When it comes to low-Reynoldsnumber high-lift airfoil design, there are three main methodologies. The Liebeck airfoils represent the first methodology, where a large rooftop suction level is used before the Stratford distribution (concave pressure recovery; see Fig. 1) [1]. This can lead to low pitching moments as well as hard-stall characteristics. The second methodology was suggested by Wortmann, where he emphasized the convex recovery and reduced any reliance on the suction peak. This methodology results in soft-stall characteristics. The last methodology, suggested by Selig and Eppler, was a compromise between the first two methodologies [7].

\section{DESIGN METHODOLOGIES}

Liebeck airfoils, such as the LNV-109A discussed here, use a large rooftop suction peak and Stratford recovery to help them achieve high $\mathrm{C} 1$, without any aft loading. The result is a very low profile-drag as well as a low lift-to-drag ratio [1]. However, this is due to the abrupt drop in $\mathrm{C} 1$ near the maximum-lift coefficient as the alpha increases, as well as the Stratford distribution. In addition, the pure Stratford Recovery airfoils are environmentally sensitive and are limited in their performance envelope. Their performance is highly affected by the Reynolds number and the flow transition location.

Initially, Wortmann proposed a distribution which consists of high aft loading with a small initial adverse pressure gradient. This results in soft-stall characteristics for this class of airfoil. As compared to other airfoils, the pressure rises with smaller gradients as the flow proceeds towards the trailing edge. Basically, the airfoil FX 63-137 (Fig. 1) was designed for human-powered aircrafts and gliders. Later, it has also been employed for other purposes.

Eppler [9] suggested that the pressure recovery should be concave; however, it is not as purely concave as proposed by Liebeck. He showed that the performance of airfoil in terms of lift coefficient can be enhanced by employing both aft loading and concave recovery collectively. Due to the aforementioned reasons, this airfoil has moderate-stall characteristics. Selig and Guglielmo [7] followed the footprints of Eppler and designed airfoil S1223 (Fig. 1), which has the highest maximum lift coefficient among all the airfoils that are presented here.

The airfoils that employ Stratford distribution (Liebeck) for pressure recovery are not the most suitable solution for motorsports applications. Dirt, moisture, and surface imperfections all act as a trip for the boundary layer. The performance of airfoils having pure concave 


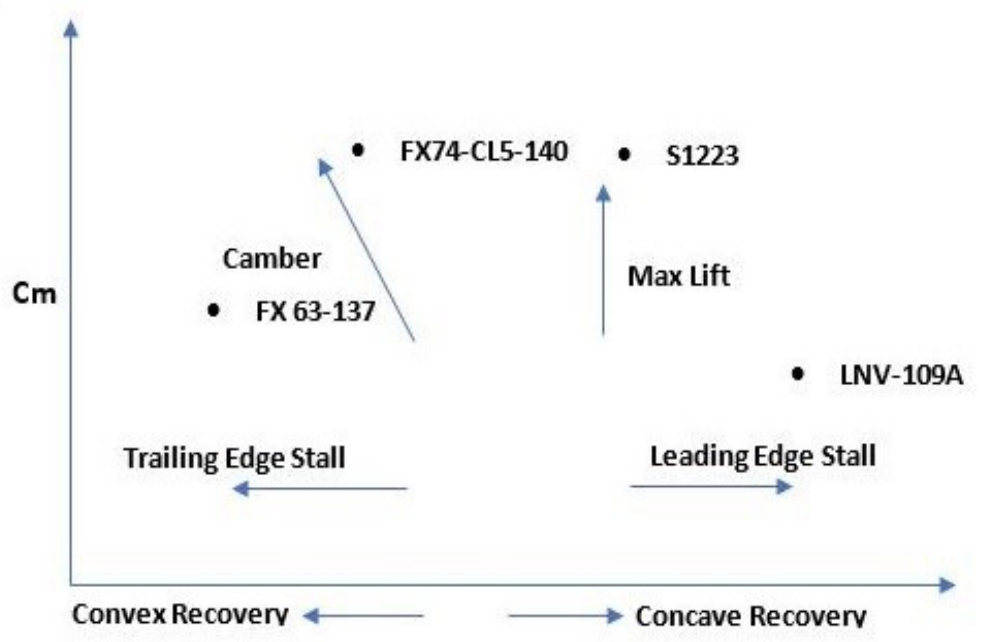

Figure 1: Low-Reynolds-number airfoil characteristics as a function of pitching-moment coefficient and upper-surface pressure recovery [7].

recovery varied depending on the transition location. The fast-moving transition point, in this case, leads to hard or leading-edge stall [7], [10]. The performance of such airfoils deteriorates rapidly in the post-stall region. So, it may have a detrimental effect on lap time if the airfoil approaches stall. Soft-stall behavior is sought in such an application as it provides flexibility in the airfoil performance near maximum-lift coefficient. This type of airfoil lift coefficient does not decrease abruptly in the post-stall region, despite a large profile drag. Moreover, it allows the aerodynamicist (a person who has expertise in aerodynamics) to choose from a wide range of angles of attack while setting up the wings on a race car depending on the nature of the race track. Race car wings have very low aspect ratios, so much of the drag comes from the induced component. Therefore, the chief concern in airfoil design is not a profile drag reduction but a high-lift coefficient [5]. Another important consideration in low-Reynolds-number airfoil design is the laminar separation bubble (LSB) and its influence on airfoil characteristics and performance.

The LSB forms when the local separation of the boundary layer occurs in the presence of an adverse pressure gradient at $\operatorname{Re} \leq 10^{6}$ (Fig. 2). After that, usually the flow makes the transition in the separated shear layer and then reattaches to the surface [1]. The profile drag of the airfoil increases and it is subjected to large variations in pressure due to the LSB. There are two types of LSB: short bubble and long bubble. It was found that the short bubble may form at a low Reynolds number. Its length and height decrease with a decrease in the Reynolds number or increase in incidence until the critical condition is achieved, known as "bursting". Increasing the Reynolds number any further either leads to the formation of long bubble or flow failure to reattach itself downstream. A very large separation bubble may cause increased profile drag, a significant decrease in lift (thin-airfoil stall), and rapid changes in the pitching moment. The chances of a thin-airfoil stall increase as the thickness/chord ratio decreases [8]. 


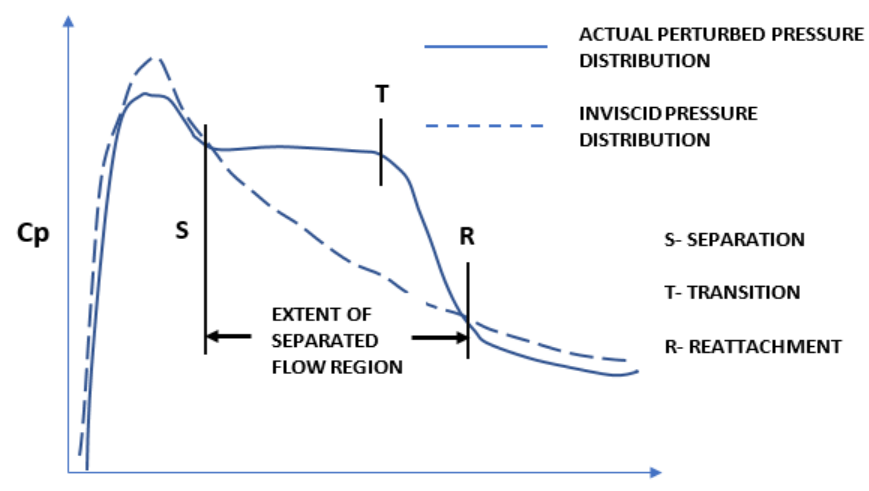

Figure 2: Chord-wise pressure coefficient distribution on the airfoil's top surface with and without a separation bubble [9].

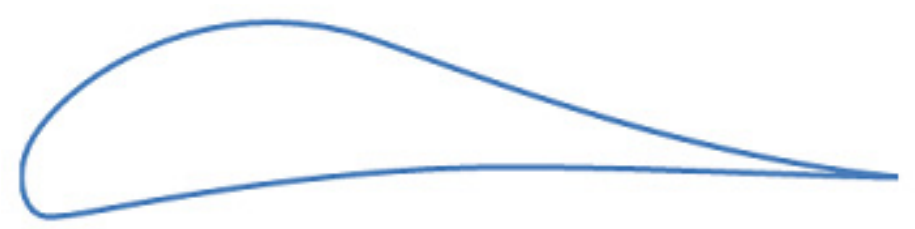

(a)

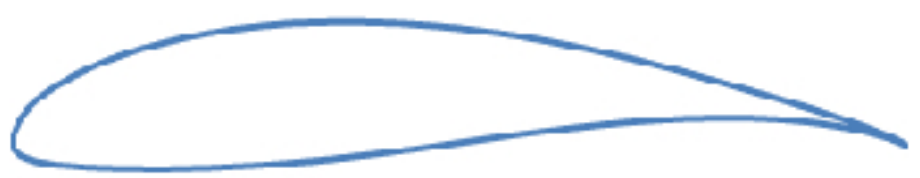

(b)

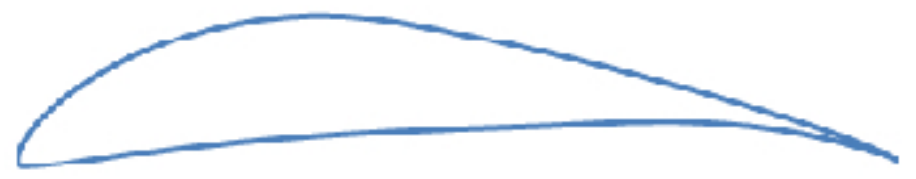

(c)

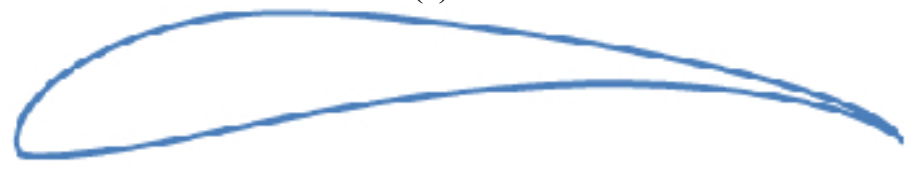

(d)

Figure 3: High-lift low-Reynolds-number airfoils used in this comparative analysis. (a) Douglas/Liebeck LNV 109A; (b) Wotmann FX 63-137; (c) Wortmann FX 74-CL5-140; (d) Selig S1223. 


\section{COMPUTATIONAL MODELING IN XFOIL}

\subsection{Methods}

The comparative study was performed using XFOIL (XFLR5 version 6.40) [10] airfoil analysis code and the airfoils' coordinates were taken from Dr. Selig's airfoil database [11]. XFOIL uses the second-order panel method to calculate the inviscid velocity distribution along the surface of the airfoil. Second-order panel methods are more accurate because of quadratically varying vorticity. The whole airfoil is divided into a finite number of vortex panels of linearly varying strength, and the flow velocity is computed along each panel after solving a set of linear algebraic equations. After that, both the pressure distribution and lift coefficient can be easily calculated [12].

\subsection{Equations}

The strength of the vortex sheet per unit length is defined in terms of distance, $\gamma(\mathrm{s})$, along the sheet:

$$
\gamma(s)=\gamma+\gamma^{\prime} s
$$

The strength of the vortex sheet depends on the difference in the tangential velocities across it:

$$
\gamma=U 1-U 2
$$

where $U 1$ and $U 2$ are the tangential velocity components.

The circulation around the vortex sheet is given by the equation,

$$
\Gamma=\int \gamma d s
$$

The lift force due to circulation is given using the Kutta-Joukowski theorem:

$$
L=\rho_{\infty} V_{\infty} \Gamma
$$

$\rho_{\infty}, V_{\infty}$ and $\Gamma$ are free stream density, free stream velocity and circulation, respectively.

The Kutta condition that must be satisfied for the existence of lift is

$$
\gamma(T E)=0
$$

The pressure coefficient can be expressed as

$$
C p=\frac{p-p_{\infty}}{\frac{1}{2} \rho V_{\infty}^{2}},
$$

where $p$ and $p_{\infty}$ are local and free stream pressures, respectively.

The section-lift coefficient is given by the following equation:

$$
C l=\frac{l}{\frac{1}{2} \rho V_{\infty}^{2} c} .
$$




\subsection{Deficiencies in modelling}

XFOIL has its limitations, like most airfoil analysis codes, and it is incapable of modeling LSBs and flow separation. It can predict stall to some extent by using empirical formulations incorporated in the code so that the maximum-lift coefficient can be estimated. Another shortcoming of XFOIL is that it assumes an instantaneous transition to turbulent flow when the trip location is specified on the airfoil surface. The prediction of transition and separation are based on the methods described by Eppler and Somers [13]. Due to these reasons, a deviation arises in the results as compared to experimental data. But, since the analysis is comparative and deviation is not very large, these findings can be used for further study.

\section{RESULTS}

Four different high-lift low-Reynolds-number airfoils were studied at $\operatorname{Re}=4 \times 10^{5}$, and their stall behavior and lift coefficients were examined. These airfoils were simulated in the aforementioned condition, with the angle of attaching varying from $-5^{\circ}$ to $20^{\circ}$ in increments of $1^{\circ}$.

From the graph plotted in Fig. 4, it has been found that both Selig S1223 and FX 74-CL5140 airfoils have the highest lift coefficient among the studied airfoils, and they also possess moderate-stall characteristics.

The pressure distributions of the studied airfoils are plotted (Figs 5-8), and it was found that the FX 63-137 (Fig. 6) has the most gradual and convex pressure recovery. Pressure is evenly distributed along the whole upper surface of the airfoil, which generally leads to trailing-edge stall at high angles of attack. This airfoil could be operated near its maximumlift coefficient region. The LNV 109A (Fig. 8) employs a high suction peak near leading edge, with concave pressure recovery to achieve a high-lift coefficient. The concave pressure recovery is undesirable in our application because it leads to the hard stall of an airfoil; that is, the airfoil is more prone to leading-edge stall. It is evident from the pressure distribution of the remaining two airfoils FX 74-CL5-140 (Fig. 7) and S1223 (Fig. 5) that both have almost moderate-stall characteristics and both airfoils also utilize an intermediate methodology, which is a mix of Wortmann and Liebeck. However, the lift coefficient of S1223 is higher than FX 74-CL5-140 (Fig. 4). Moreover, the S1223 also possesses more favourable stall characteristics relative to FX 74-Cl5-140.

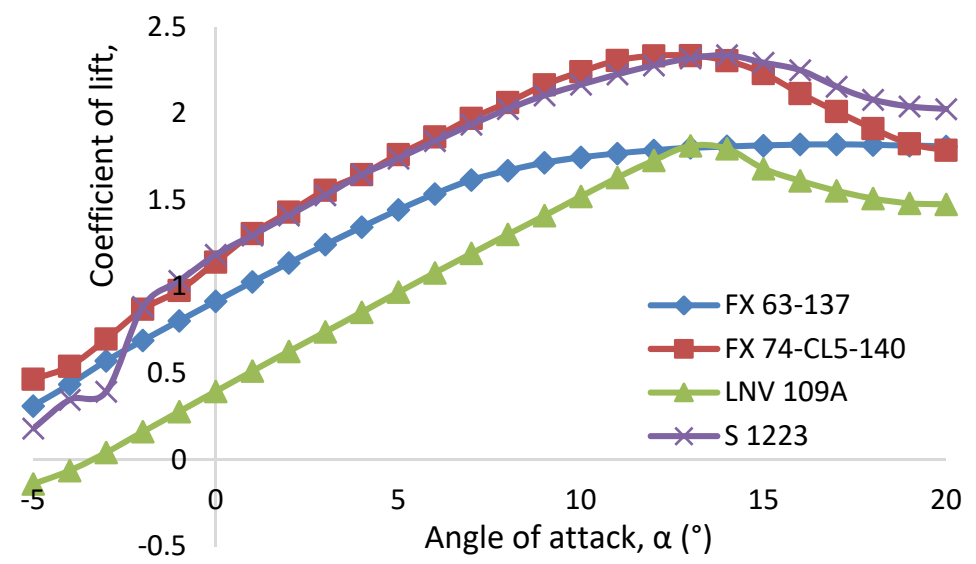

Figure 4: $\mathrm{Cl}$ versus $\alpha$ comparison of four selected airfoils at $\mathrm{Re}=4 \times 10^{5}$. 


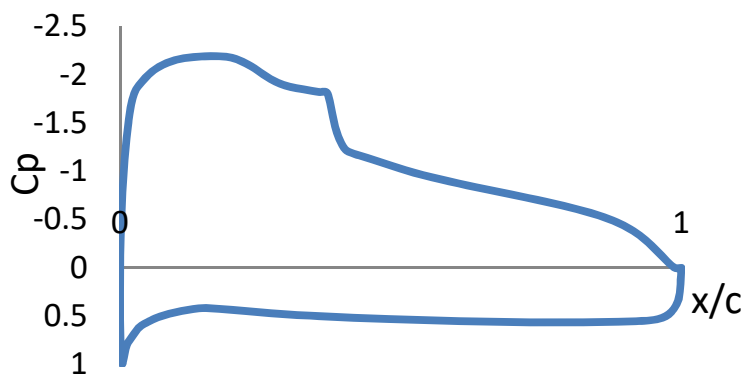

Figure 5: Pressure distribution of S1223 airfoil at $\alpha=5^{\circ}$ at $\mathrm{Re}=4 \times 10^{5}$ (free transition).

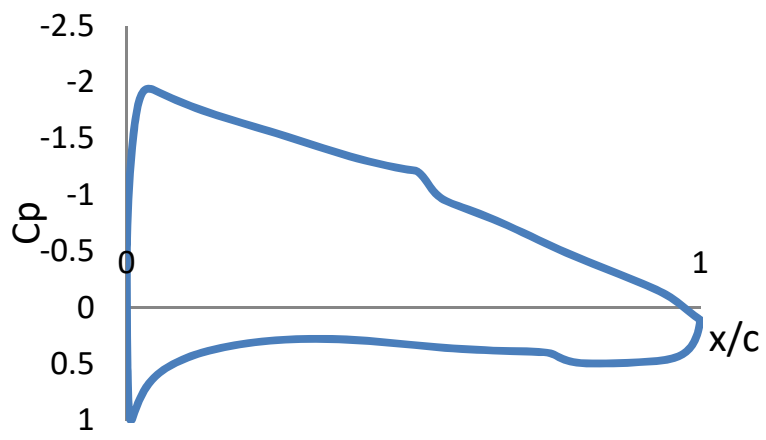

Figure 6: Pressure distribution of FX 63-137 airfoil at $\alpha=5^{\circ}$ at $\mathrm{Re}=4 \times 10^{5}$ (free transition).

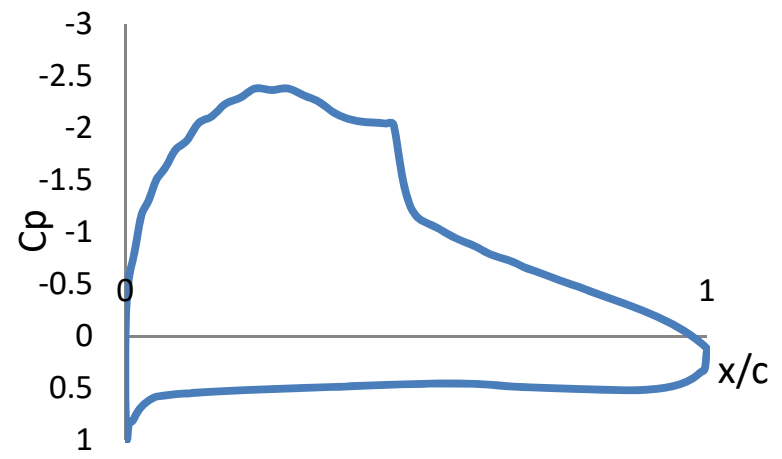

Figure 7: Pressure distribution of FX 74-CL5-140 airfoil at $\alpha=5^{\circ}$ at $\operatorname{Re}=4 \times 10^{5}$ (free transition). 


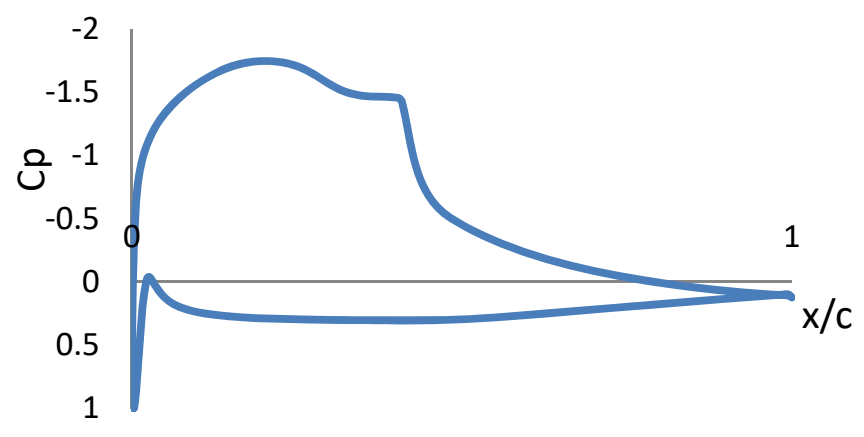

Figure 8: Pressure distribution of LNV 109A airfoil at $\alpha=5^{\circ}$ at $\operatorname{Re}=4 \times 10^{5}$ (free transition).

As the race car wings are operating close to the ground, there is always a strong possibility that the surface imperfections, dirt, and other particles may act as a trip element and modify the flow nature. The performance of high-lift low-Reynolds-number airfoils also depends heavily on the flow transition location. This can be observed from the graphs plotted using XFOIL. Almost all airfoils have shown large performance variations when the boundary layer has been tripped on the upper airfoil surface.

Fig. 9 depicts a comparison of $\mathrm{Cl}$ vs $\alpha$ polars of the LNV 109A airfoil in the case of free transition and when the transition is forced at $10 \%$ of the chord length. A large variation in lift coefficient is observed in the range of 10-20 degrees $\alpha$ and the curve almost flattens in case of the tripped boundary layer. The maximum-lift coefficient of LNV 109A drastically decreases when the boundary layer is tripped on the top surface of the airfoil at $10 \%$ of the chord length. The performance of all high-lift airfoils degrades in the presence of a tripping element.

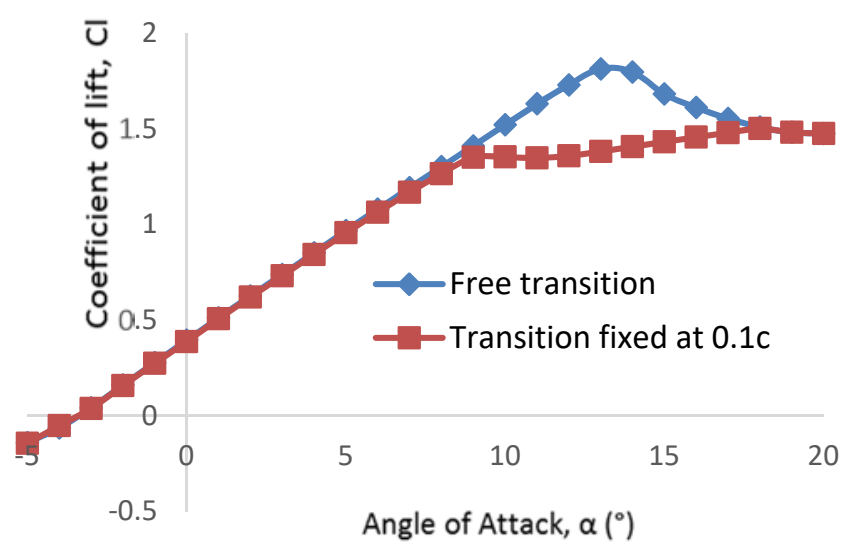

Figure 9: Comparison of $\mathrm{Cl}$ vs $\alpha$ polars of the LNV 109A airfoil at $\mathrm{Re}=4 \times 10^{5}$ in the case of free transition and transition fixed at $10 \%$ of the chord. 
Fig. 10 shows that FX 63-137 has lower values of lift coefficient when the alpha ranges from -5 to 13 degrees in the fixed-transition case; however, both the curves level out at approximately 15 degrees of alpha. Overall, the graph illustrates similar trends in both cases.

FX 74-CL5-140 shows huge variation between the forced transition and free transition (Fig. 11). It has had performance almost comparable to $\mathrm{S} 1223$ in the case of free transition, but there is a large discrepancy in a forced transition scenario.

The S1223 in both cases depicts a similar trend, with a high-lift coefficient and moderatestall characteristics (Fig. 12). In comparison to the remaining airfoils under consideration, the maximum-lift coefficient of the S1223 is found to be much higher when the transition is forced at $10 \%$ of the chord length at the top surface. So, after considering a number of aspects, it is concluded that S1223 is the most viable choice among these airfoils.

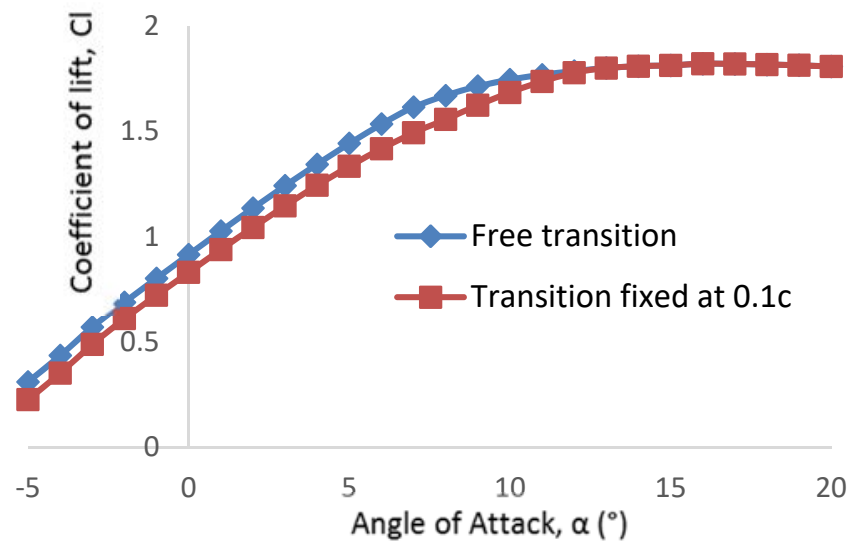

Figure 10: Comparison of $\mathrm{Cl}$ vs $\alpha$ polar of $\mathrm{FX} \mathrm{63-137} \mathrm{airfoil} \mathrm{at} \mathrm{Re}=4 \times 10^{5}$ in the case of free transition and transition fixed at $10 \%$ of the chord.

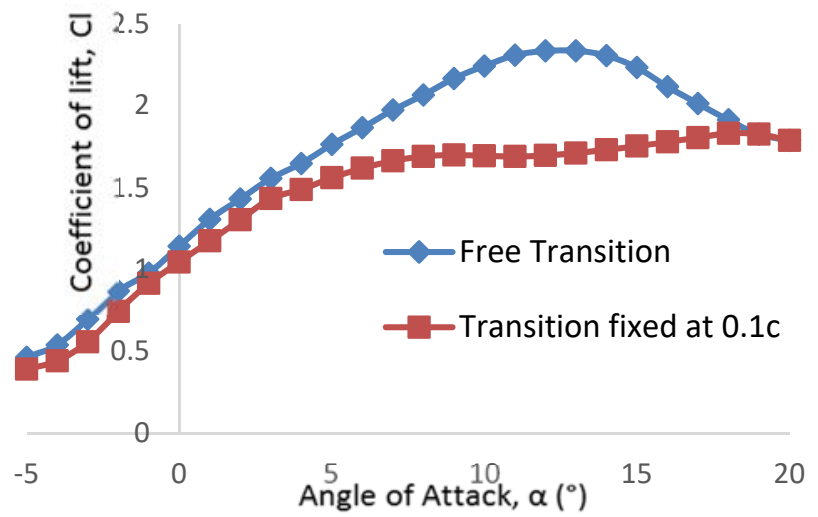

Figure 11: Comparison of Cl vs $\alpha$ polar of FX 74-CL5-140 airfoil at $\mathrm{Re}=4 \times 10^{5}$ in the case of free transition and transition fixed at $10 \%$ of the chord. 


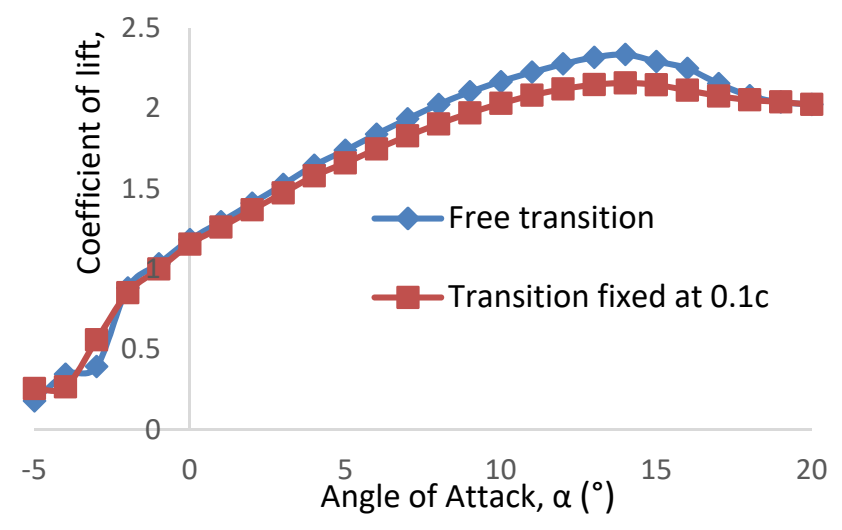

Figure 12: Comparison of $\mathrm{Cl}$ vs $\alpha$ polar of $\mathrm{S} 1223$ airfoil at $\mathrm{Re}=4 \times 10^{5}$ in the case of free transition and transition fixed at $10 \%$ of the chord.

\section{CONCLUSION}

The analysis and comparison of $\mathrm{Cl}$ versus $\alpha$ (in both free- and forced-transition cases) and surface pressure distribution plots reveals that the Selig S1223 airfoil is the most suitable airfoil. Despite many performance similarities with FX 74-CL5-140, S1223 is found to be superior overall. The variation in performance of S1223 is also lower as compared to the remaining considered airfoils, which is evident in the case of forced transition. The results of this paper are helpful in selecting the airfoil for the front/rear wings of team Formula Fusion's FS vehicle of the NED University of Engineering and Technology. As multi-element airfoils are employed in FS vehicles, the future study will focus on the arrangement of S1223 in the multi-element configuration for high downforce.

\section{ACKNOWLEDGEMENTS}

The authors would like to thank Ahmet Ergin Efendioglu (Turkish Petroleum Corporation) and Ms. Fizza Naqvi (Petroleum Engineering department, NED University of Engineering and Technology) for their unconditional support.

\section{REFERENCES}

[1] Gad-el-Hak, Control of low-speed airfoil aerodynamics. AIAA, 28(9), 1990.

[2] Craig, C. \& Passmore, M.A., Methodology for the design of the aerodynamic package for a Formula SAE vehicle. SAE Int J Passenger Cars - Mech Systems, 7(2), pp. 575-585, 2014.

[3] F1, Aerodynamics. www.formula1.com/en/championship/inside-f1/understanding-f1racing/Aerodynamics.html.

[4] Fuller, M.J., A brief history of sports car racing. www.mulsannescorner.com/ history.html.

[5] Liebeck, R., Subsonic airfoil design. Applied Computational Aerodynamics, Vol. 125, ed. P.A. Henne, American Institute of Aeronautics and Astronautics: Washington, DC, pp. 501-530, 1990.

[6] Katz, J., Race Car Aerodynamics, Robert Bentley Publishers: Cambridge, MA, 1995. 
[7] Selig, M.S. \& Guglielmo, J.J., High-lift low Reynolds number airfoil design. J. Aircraft, 34(1), pp. 72-79, 1997.

[8] Jahanmiri, M., Laminar separation bubble: Its structure, dynamics, and control. Division of Fluid Dynamics, Department of Applied Mechanics, Chalmers University of Technology, 2011.

[9] Eppler, R., Turbulent airfoils for general aviation. J. Aircraft, 15(2), pp. 93-99, 1978.

[10] Drela, M., XFOIL: An analysis and design system for low Reynolds number airfoils. Low Reynolds Number Aerodynamics, Springer: Berlin, Heidelberg, pp. 1-12, 1989.

[11] UIUC Applied Aerodynamics Group, UIUC Airfoil Coordinates Database. http://mselig.ae.illinois.edu/ads/coord_database.html.

[12] Katz, J. \& Plotkin, A., Low-Speed Aerodynamics, McGraw-Hill, 1991.

[13] Eppler, R. \& Somers, D., A computer program for the design and analysis of low-speed airfoils. NASA TM-80210, 1980. 